\title{
Management of pyogenic vertebral osteomyelitis with spinal cord compression in the elderly
}

\author{
R Gepstein, ${ }^{1}$ Y Folman, ${ }^{2}$ C Lidor,${ }^{1}$ V Barchilon, ${ }^{1}$ A Catz, ${ }^{3}$ T Hallel ${ }^{1}$ \\ ${ }^{1}$ Department of Orthopaedics, Meir Hospital, Kfar Saba 44281, Israel; ${ }^{2}$ Department of \\ Orthopaedics, Hillel-Jaffe Hospital, Hadera, Israel; ${ }^{3}$ Loewenstein Rehabilitation Hospital, \\ Raanana, Israel.
}

Five elderly and debilitated patients presented with compressive myelopathy due to pyogenic spondylitis. All had undergone surgery which consisted of eradication of the infected tissue via an anterior approach followed by primary bone graft. Supplementary antibiotic treatment was determined by intraoperative bacteriological culture. This aggressive approach, disregarding the patients' advanced age and poor general medical state, resulted in total resolution of the neurological deficit and the patients' return to their preoperative everyday activity.

\section{Keywords: pyogenic spondylitis in elderly; spinal cord compression; anterior spinal surgery.}

\section{Introduction}

The distribution of osteomyelitis shifts with age from the appendicular skeleton to the spinal column where it most commonly affects the vertebral bodies.

While there is no doubt that vertebral osteomyelitis may be treated conservatively with success, ${ }^{1,2}$ impairment to the point of paralysis must be surgically treated. ${ }^{3-5}$ This surgical treatment, ie decompression of the spinal cord, can be achieved in various ways. Posterial approach via laminectomy was rejected due to its destabilising affect on that spine which had already been affected in its anterior column. ${ }^{3,6.7}$ Costotransversectomy did enable drainage of the abscess but no more and as neurological recovery demands total elimination of the necrotic tissue this approach had limited effects. ${ }^{3}$

Therefore, anterior surgery was adopted, namely extensive corpectomy via a transthoracic approach. This was supplemented with parenteral antibiotics for a minimum of 6 weeks as determined by the intraoperative culture.

The following is a report on our experience with 5 elderly patients with a mean age of 70 years treated in this manner.

\section{Patients and methods}

From 1986 to 19895 elderly and debilitated patients were treated for haematogenous pyogenic vertebral osteomyelitis. They were 3 females and 2 males with a mean age of 70 years (range 67-75). Three patients had a past medical history of diabetes mellitus. All the 5 lesions affected the thoracic spine. The patients were operated on an emergency basis due to increasing neurological deficit. Surgery was performed via a left thoracotomy; granulation tissue, necrotic disc and bone were curetted back to bleeding cancellous bone. Autogenous tricortical iliac strut was grafted. Antibiotic treatment as determined by intraoperative culture results was administered postoperatively for 6 weeks. The patients used a removable brace for immobilisation. Medical records and roentgenograms of the hospitalisation and outpatient follow up served as the basis of this retrospective study.

\section{Results}

Three cultures grew gram-positive cocci (Staphylococcus aureus) and two gramnegative bacilli (Escherichia coli and Klebsiella). Pathology reports were consistent with chronic osteomyelitis. The source of the infecting organism was not identified. The onset of symptoms was abrupt. Spontaneous back pain was followed by increasing weakness in the legs, in 2 cases combined with urinary incontinence. The average time from the inception of symptoms to 
diagnosis was 10 weeks (range 3-12 weeks). Clinical examination revealed local tenderness about the affected vertebrae and different grades of paraparesis (Frankel class $\mathrm{C}$ or D). Low grade fever was present in 2 patients. Laboratory studies showed consistent highly elevated erythrocyte sedimentation rate. All the patients had bone scan and plain film roentgenograms positive for a vertebral lesion. All had computed tomography and 2 had an additional myelogram that showed an extradural spinal cord compression. Clinical follow up averaged 29 months (25 to 33 months). All patients showed complete resolution of the paralysis (Frankel class E) within 3-6 months. Three patients showed mild symptoms related to surgery. Radiographic follow up, up to 12 months, including tomograms, showed incorporation of the grafts into the adjacent vertebral bodies.

\section{Illustrative case report}

A 74 year old woman presented with a 2-week history of severe upper back pain and gait difficulty. The symptoms appeared shortly after the patient underwent a thoracotomy and lobectomy for a lung abscess which cultured a Gram-negative Klebsiella bacilli. When seen by us the patient was being treated with Ceftriaxone (Rocephin) in daily doses of 2 grams. Clinical examination showed local tenderness over the thoracic spine, and mild signs of upper motor neuron paraparesis (Frankel class D). The laboratory profile was normal except for a markedly elevated erythrocyte sedimentation rate. The bone scan revealed a solitary increased uptake over the T6 and T7 vertebrae. Roentgenograms revealed narrowing of the T6-7 disc space with erosion of the end plates (Fig 1). A postmyelogram computed tomogram showed extradural compression of the thecal sac. Surgery consisted of an anterior spinal decompression, excision of the necrotic vertebrae and stabilisation with 3 strut grafts cut from the resected rib. While the presumptive diagnosis of Klebsiella infection was considered, Staphylococcus aureus was identified from cultures. The patient was given a 6-week course of parental cloxacillin sodium (Or-

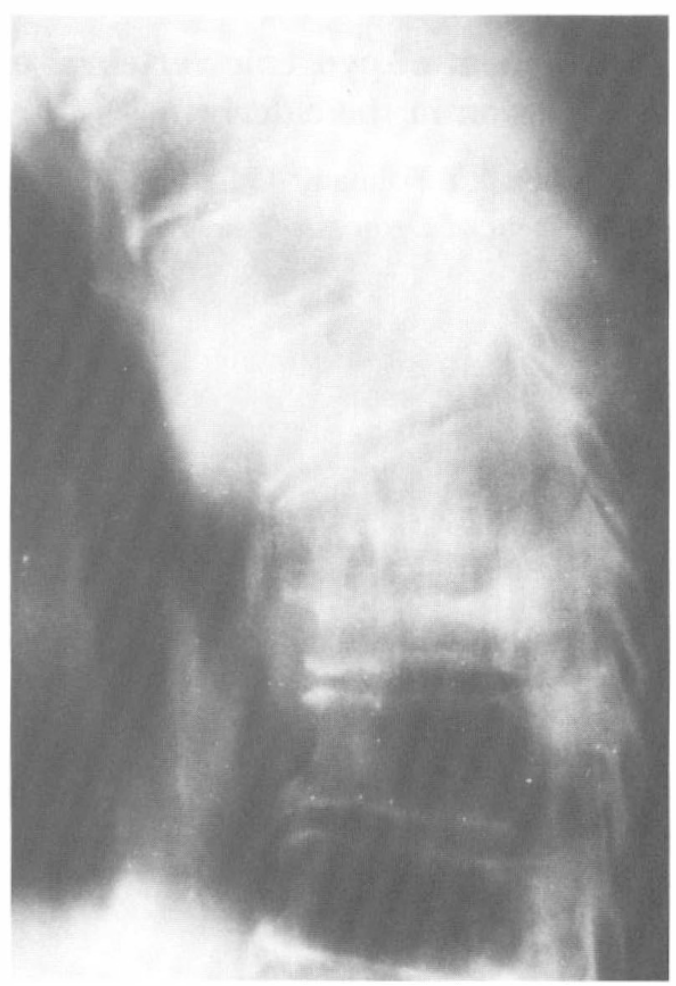

Figure 1 Osteomyelitis of T6-T7. Note blurring of end plates.

benin) in daily doses of 4 grams. By the time of discharge, the patient was pain-free and fully ambulatory (Frankel class E) wearing a Jewett-type brace. At follow up examination 24 months postoperatively, the patient was continuing to do well, and tomographs showed incorporation of the graft into the adjacent vertebral bodies (Fig 2).

\section{Discussion}

The most common mechanism of pyogenic vertebral osteomyelitis is a blood-borne metastasis. ${ }^{2.8}$ The patient presented as the illustrative case was, with reason, thought to be suffering a similar syndrome. The culture report, which came as a surprise to us, emphasised the importance of open biopsy. The clinical presentation in the elderly may be atypical and vague, leading to delay in diagnosis. ${ }^{5,7.9-12}$ In fact, all 5 patients were referred to us with progressive paralysis, the elderly signs having been largely ignored. 


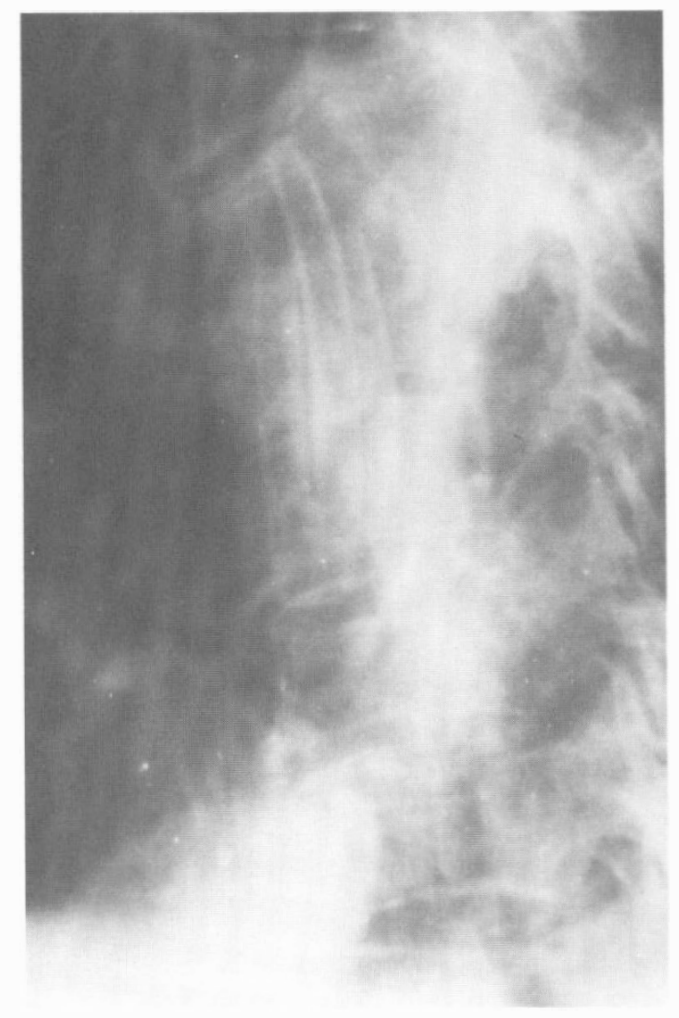

Figure 2 Postoperative $x$-rays. The bone graft (ribs) in place.

Elderly patients with acute back pain and local tenderness over the spine should be strongly suspected of having vertebral osteomyelitis. Increased erythrocyte sedimentation rate is the most consistent laboratory study to support the diagnosis and to monitor the course of infection. ${ }^{1,2}$ Leucocytosis is not a constant finding. ${ }^{2.5}$ As in the above cases, bone scanning may localise the lesion at an early phase while roentgenographic changes are not detectable for at least 8 to 12 weeks after the onset of symptoms. ${ }^{1,2}$ Myelography may show the extent of the epidural abscess, ${ }^{11,13}$ but more recent noninvasive MRI became the study of choice. As the diagnosis data is nonspecific, it is necessary to approach the lesion directly in order to obtain tissue for histological and bacteriological examination. In cases such as the above, the paralysis itself justified immediate operative decompression, while the laboratory examinations were performed postoperatively. This is contrary to the accepted approach in nonparalysed patients of establishing a definitive diagnosis prior to treatment. The main differential diagnosis includes tuberculous and fungal infections, primary and metastatic tumours. The instability caused by destruction of bony structures, the direct pressure from epidural extension of the abscess and septic thrombosis of the spinal vascular plexus may all cause progressive paralysis. ${ }^{14}$ Eismont $^{3}$ pointed out that increased age, the associated disease of diabetes mellitus, a more cephalad level of infection and the presence of Staphylococcus aureus predispose to paralysis. Our patients frequently presented this deleterious combination. The results of the treatment as presented were superior for spinal osteomyelitis to those accepted for an infected apendicular skeleton, not only because the anatomy of the spine enables extensive removal of the affected segment, but also because of the local vascularity. While the rich blood supply is the predisposing factor for the high predominance of infection in the vertebrae, this same rich blood supply contributes to the efficient incorporation of the graft and of high levels of antibiotics at the site of infection. The high probability of cure justifies this seemingly aggressive surgical and medical approach disregarding the advanced age and poor general health of the patient.

\section{References}

1 Digby JM, Kersley JB (1979) Pyogenic non-tuberculous spinal infection. An analysis of thirty cases. J Bone Joint Surg 61: 47-55.

2 Ross PM, Fleming JL (1976) Vertebral body osteomyelitis. Clin Orthop 118: 190-198.

3 Eismont FJ, Bohlman HH, Soni PL, Goldberg VM, Freehafer AA (1983) Pyogenic and fungal vertebral osteomyelitis with paralysis. J Bone Joint Surg 65A: 19-29.

4 Emery SE, Chan DPK, Woodward HR (1989) Treatment of hematogenous pyogenic vertebral osteomyelitis with anterior debridement and primary bone grafting. Spine 14: 284-291.

5 Joughlin E, McDougall C, Parfitt C, Yong-Hing K, Kirkaldy-Willes WH (1991) Causes and clinical management of vertebral osteomyelitis in Saskatchewan. Spine 16: 262-264. 
6 Fellander MAC (1975) Paraplegia in spondylitis: Results of operative treatment. Paraplegia 13: 75-88.

7 Kemp HB, Jackson JW, Shaw NC (1974) Laminectomy in paraplegia due to infective spondylosis. $B r J$ Surg 61: 66-72.

8 Henson SW, Coventry MB (1956) Osteomyelitis of the vertebrae as the result of infection in the urinary tract. Surg Gynecol Obstet 102: 207-214.

9 Garcia A, Grantham SA (1960) Hematogenous pyogenic vertebral osteomyelitis. J Bone Joint Surg 42A: 429-436.

10 Griffiths HED, Jones DM (1971) Pyogenic infection of the spine. J Bone Joint Surg 538: 383-391.

11 Lifeso RM (1990) Pyogenic spinal sepsis in adults. Spine 15: 1265-1271.

12 Wedge IH, Oryschak AF, Robertson DE, Kirkaldy-Willis WH (1977) Atypical manifestations of spinal infection. Clin Orthop 123: 155-163.

13 Chappel R, Verhelst JA, Nagler JM, Dom L, Appel B, Herregods P (1986) Epidural abscess causing tetraparesis: Case report. Paraplegia 24: 364-369.

14 Kulowski J (1936) Pyogenic osteomyelitis of the spine. An analysis and discussion of 102 cases. J Bone Joint Surg 18: 343-364. 